\title{
A REDUCTION OF ALGEBRAIC REPRESENTATIONS OF MATROIDS
}

\author{
BERNT LINDSTROัM
}

\begin{abstract}
We prove the following result conjectured by M. J. Piff in his thesis (1972).

THEOREM. Let $M$ be a matroid with an algebraic representation over a field $F(t)$, where $t$ is transcendental over $F$. Then $M$ has an algebraic representation over $F$.

The proof depends on Noether's normalization theorem and the place extension theorem. We obtain the following corollary.

COROLlaRY. If a matroid is algebraic over a field $F$, then any minor of $M$ is algebraic over $F$.
\end{abstract}

We will assume that the reader is familiar with the foundations of matroid theory (cf. [3]). A matroid is called algebraic over a field when there is an algebraic representation of $M$ over this field [3, p. 184]. M. J. Piff proved in his thesis [2, Theorem 3.3], with the aid of the lemma below, that a matroid $M$, which is algebraic over a field $F$ with prime field $P$, is algebraic over a transcendental extension $P\left(t_{1}, \ldots, t_{m}\right)$ of $P$. He conjectured that $M$ is algebraic over $P$. This is true by our theorem.

THEOREM. Let the matroid $M$ be algebraic over a transcendental extension $F(t)$ of $F$. Then $M$ is algebraic over $F$.

We will apply the following lemma [2, Lemma 3.2], which is not hard to prove.

LEMMA. If $M$ is algebraic over $F(\alpha)$, where $\alpha$ is algebraic over $F$, then $M$ is algebraic over $F$.

Proof of THE THEOREM. Let $M$ be an algebraic matroid with elements $E$, a finite subset of a field $K . M$ is assumed to be algebraic over $F(t)$, a subfield of $K$ with $t$ transcendent over $F$. The transcendence degree of $F(E)$ over $F(t)$ equals $r$, the rank of $M$. Let $E=\left\{e_{1}, \ldots, e_{n}\right\}$.

By Noether's normalization theorem [1, Chapter II, Theorem 1] there are elements $y_{1}, \ldots, y_{r}$ of $K$ such that each $e_{i}(1 \leqslant i \leqslant n)$ is integral over $F(t)\left[y_{1}, \ldots, y_{r}\right.$. Let $E^{\prime}=E \cup\left\{y_{1}, \ldots, y_{r}\right\}$ and let $M^{\prime}$ denote the algebraic matroid of $M^{\prime}$ over $F(t)$. The matroid $M^{\prime}$ is an extension of $E^{\prime}$. Let $\mathscr{C}$ be the set of all circuits of $M^{\prime}$.

We associate variables $X_{i}(1 \leqslant i \leqslant n+r)$ to the elements of $E^{\prime}$ such that $e_{i} \leftrightarrow X_{i}$ for $1 \leqslant i \leqslant n$ and $y_{j} \leftrightarrow X_{n+j}$ for $1 \leqslant j \leqslant r$. When $A \subseteq E^{\prime}$ let $X_{A}$ denote the

Received by the editors February 10, 1986.

1980 Mathematics Subject Classification (1985 Revision). Primary 05B35, 12 F99.

This research was supported by the Swedish Natural Science Research Council. 
variables $X_{k}$ corresponding to the elements of $A$ in some arbitrarily fixed order. For each circuit $C \in \mathscr{C}$ there is an irreducible polynomial $P_{C}\left(X_{C}\right)$ over $F[t]$ satisfied by $C$, i.e. $P_{C}(C)=0$. All the variables $X_{i}$ of $X_{C}$ occur explicitely in $P_{C}\left(X_{C}\right)$ since $C$ is a circuit.

Let $\bar{F}$ and $\bar{K}$ be the algebraic closures of $F$ and $K$ respectively. We will obtain a ring homomorphism

$$
\varphi: F\left[t, y_{1}, \ldots, y_{r}\right] \rightarrow \bar{F}\left[y_{1}, \ldots, y_{r}\right] .
$$

Define $\varphi\left(y_{i}\right)=y_{i}$ for $1 \leqslant i \leqslant r$. Then choose $\varphi(t) \in \bar{F}$ distinct from all zeros of coefficients of the polynomials $P_{C}, C \in \mathscr{C}$ (by assumption the coefficients belong to $F[t])$. This $\varphi(t)$ can be found since the number of zeros is finite and $\bar{F}$ is infinite. Then let $\varphi(a)=a$ for $a \in F$ and extend $\varphi$ to the ring homomorphism $\varphi$ : $F\left[t, y_{1}, \ldots, y_{r}\right] \rightarrow \bar{F}\left[y, \ldots, y_{r}\right],\left(t, y_{1}, \ldots, y_{r}\right.$ are algebraically independent over $\left.F\right)$.

Note that $\left\{y_{1}, \ldots, y_{r}\right\}$ is a base of $M^{\prime}$ since $e_{1}, \ldots, e_{n}$ are algebraic over $F(t)\left[y_{1}, \ldots, y_{r}\right]$ and the transcendence degree of $\left\{e_{1}, \ldots, e_{n}\right\}$ over $F(t)$ is $r$.

By the place extension theorem [1, Chapter I, Theorem 1] we may extend $\varphi$ to a place $\varphi: \bar{K} \rightarrow \bar{K} \cup\{\infty\}$. Since $e_{i} \in E$ is integral over $F(t)\left[y_{1}, \ldots, y_{r}\right]$ it follows that $\varphi\left(e_{i}\right)$ is finite [1, Chapter I, Proposition 4]. We shall prove that the restriction of $\varphi$ to $E$ gives an algebraic representation of $M$ over $\bar{F}$. The existence of an algebraic representation over $F$ follows then by the lemma.

Now let $P_{C}^{\varphi}\left(X_{C}\right) \in \bar{F}\left(X_{C}\right)$ be the polynomial obtained by applying $\varphi$ to the coefficients of $P_{C}$. Note that the polynomial $P_{C}^{\varphi}$ is nonzero by the choice of $\varphi(t)$. Since $P_{C}^{\varphi}(\varphi(C))=0$ it follows that dependent subsets $A \subseteq E$ have images $\varphi(A)$ which are algebraically dependent over $\bar{F}$.

Then let $B$ be a base of $M$. We shall prove that $\varphi(B)$ has transcendence degree $r$ over $\bar{F}$. Let $C_{i}$ be the fundamental circuit of $y_{i}$ with respect to the base $B$ and let $P_{C_{i}}\left(X_{C_{i}}\right)$ be the associated polynomial. Since $P_{C_{i}}^{\varphi}\left(X_{C_{i}}\right)$ contains all variables explicitly and $P_{C_{i}}^{\varphi}\left(\varphi\left(C_{i}\right)\right)=0$, it follows that $y_{i}\left(=\varphi\left(y_{i}\right)\right)$ is algebraic over $\bar{F}(\varphi(B))$. Since $y_{1}, \ldots, y_{r}$ the algebraically independent over $F$, we conclude that $\varphi(B)$ has transcendence degree $r$ over $\bar{F}$.

Therefore $\varphi: E \rightarrow \bar{K}$ is an algebraic representation of $M$ over the field generated by the coefficients of the polynomials $P_{C}^{\varphi}$, a field which is finitely generated algebraic over $F$. Repeated application of the lemma gives then an algebraic representation over $F$, and the theorem is proved.

COROLlaRY. If a matroid $M$ is algebraic over a field $F$, then any minor of $M$ is algebraic over $F$.

Proof. By [3, Theorem 11.3.1] a minor of $M$ is algebraic over some transcendental extension of $F$. The result follows then by our theorem.

\section{REFERENCES}

1. S. Lang, Introduction to algebraic geometry, Interscience, New York, 1958.

2. M. J. Piff, Some problems in combinatorial theory, Ph.D. thesis, Oxford, 1972.

3. D. J. A. Welsh, Matroid theory, Academic Press, London, 1976.

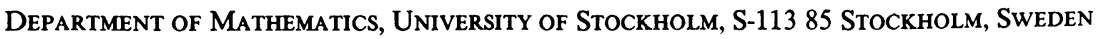

